\title{
Teaching Video NeuroImages: Multisystemic Erdheim-Chester Disease Presenting as a Cerebellar Ataxia
}

Marcelo Houat de Brito, MD, Marcos Castello Barbosa de Oliveira, MD, Yuri Reis Casal, MD, Andre Neder Ramires Abdo, MD, Leandro Tavares Lucato, MD, PhD, and Mateus Mistieri Simabukuro, MD Neurology ${ }^{\circledR}$ 2021;96:e3005-e3006. doi:10.1212/WNL.0000000000011714

Figure 1 Brain MRI and Chest/Abdomen/Pelvis CT
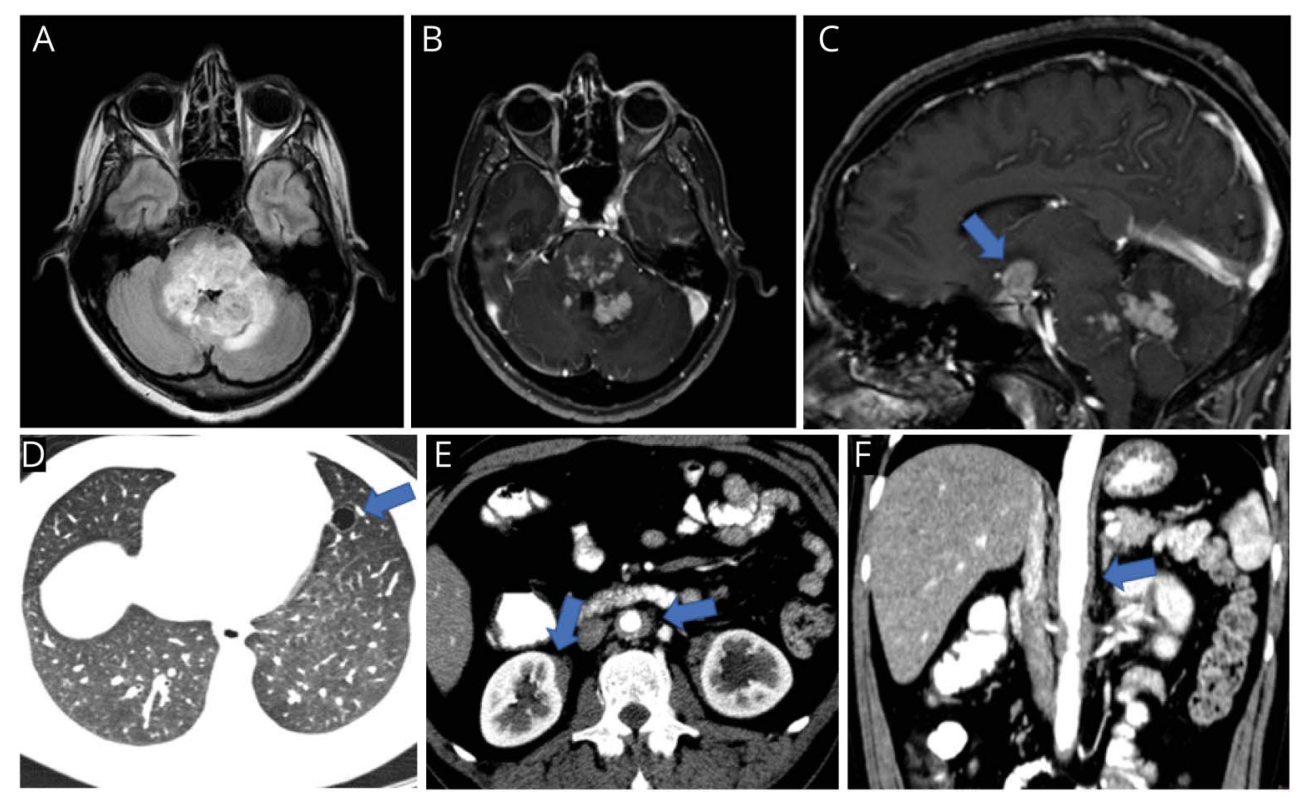

Brain MRI demonstrates infiltrative lesion in the pons and cerebellar peduncles: (A) fluid-attenuated inversion recovery hyperintense and (B, C) T1/gadolinium-enhancing. An enhancing pituitary lesion was also present (arrow in C). $\mathrm{CT}$ images demonstrate a pulmonary cyst and posterior ground-glass pattern (D) and perirenal and periaortic soft tissue $(E, F)$.

A 41-year-old man was admitted to the neurology ward due to progressive vertigo and unsteadiness for the previous 2 months. Neurologic examination was remarkable for a global cerebellar syndrome. Investigation with brain MRI led to the hypothesis of a histiocytosis due to infiltrative lesions of the pons, cerebellar peduncles, and pituitary. Therefore, investigation progressed with chest/abdomen/pelvis CT, bone scintigraphy, and a tibial biopsy that confirmed the diagnosis of Erdheim-Chester disease (figures 1 and 2, video).

Erdheim-Chester disease is a rare disorder characterized by the infiltration of non-Langerhans histiocytes in multiple tissues, mainly bone, but with CNS involvement in around $40 \%$ of cases. $^{1,2}$

\section{Study Funding}

No targeted funding reported.

\section{Correspondence}

Dr. de Brito

marcelohbrito@gmail.com
MORE ONLINE

- Video

$\rightarrow$ Teaching slides

links.lww.com/WNL/

B336

From the Departments of Neurology (M.H.d.B., M.C.B.d.O., M.M.S.), Pathology (Y.R.C.), and Radiology (L.T.L.), Faculdade de Medicina, Hospital das Clínicas, Universidade de São Paulo; Instituto do Câncer do Estado de São Paulo (M.H.d.B., M.C.B.d.O., A.N.R.A.), Brazil.

Go to Neurology.org/N for full disclosures. Funding information and disclosures deemed relevant by the authors, if any, are provided at the end of the article. 

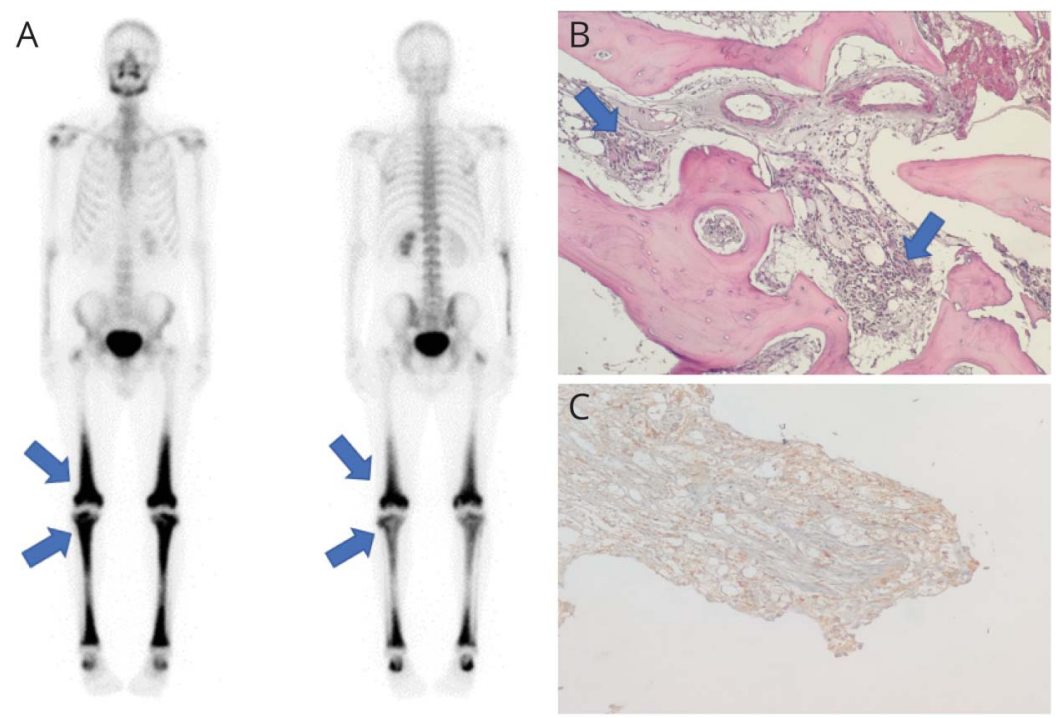

(A) Bone scintigraphy with technetium shows increased concentration in long bones (arrows in proximal tibia and distal femur, common disease locations). (B) H\&E of the tibia biopsy (100x) reveals intense chronic lymphohistiocytic inflammatory infiltrate in between intact bone trabeculae (arrows). (C) CD68 immunostain of the tibia biopsy (100x) confirms positive histiocytes (red).

\section{Disclosure}

The authors report no disclosure relevant to the manuscript. Go to Neurology.org/N for full disclosures.

\section{Appendix Authors}

\begin{tabular}{|c|c|c|}
\hline Name & Location & Contribution \\
\hline $\begin{array}{l}\text { Marcelo } \\
\text { Houat de } \\
\text { Brito, MD }\end{array}$ & $\begin{array}{l}\text { Universidade de São } \\
\text { Paulo, Faculdade de } \\
\text { Medicina, Hospital das } \\
\text { Clínicas }\end{array}$ & $\begin{array}{l}\text { Design and } \\
\text { conceptualization of the } \\
\text { study; data collection, } \\
\text { analysis, and } \\
\text { interpretation; drafting } \\
\text { the manuscript }\end{array}$ \\
\hline $\begin{array}{l}\text { Marcos } \\
\text { Castello } \\
\text { Barbosa de } \\
\text { Oliveira, MD }\end{array}$ & $\begin{array}{l}\text { Universidade de São } \\
\text { Paulo, Faculdade de } \\
\text { Medicina, Hospital das } \\
\text { Clínicas }\end{array}$ & $\begin{array}{l}\text { Data collection, analysis, } \\
\text { and interpretation; } \\
\text { revision of manuscript }\end{array}$ \\
\hline $\begin{array}{l}\text { Yuri Reis } \\
\text { Casal, MD }\end{array}$ & $\begin{array}{l}\text { Universidade de São } \\
\text { Paulo, Faculdade de } \\
\text { Medicina, Hospital das } \\
\text { Clínicas }\end{array}$ & $\begin{array}{l}\text { Data collection, analysis, } \\
\text { and interpretation }\end{array}$ \\
\hline
\end{tabular}

Appendix (continued)

\begin{tabular}{|c|c|c|}
\hline Name & Location & Contribution \\
\hline $\begin{array}{l}\text { Andre Neder } \\
\text { Ramires Abdo, } \\
\text { MD }\end{array}$ & $\begin{array}{l}\text { Universidade de São } \\
\text { Paulo, Faculdade de } \\
\text { Medicina, Hospital das } \\
\text { Clínicas }\end{array}$ & $\begin{array}{l}\text { Data collection, analysis, } \\
\text { and interpretation; } \\
\text { revision of the } \\
\text { manuscript }\end{array}$ \\
\hline $\begin{array}{l}\text { Leandro } \\
\text { Tavares } \\
\text { Lucato, MD, } \\
\text { PhD }\end{array}$ & $\begin{array}{l}\text { Universidade de São } \\
\text { Paulo, Faculdade de } \\
\text { Medicina, Hospital das } \\
\text { Clínicas }\end{array}$ & $\begin{array}{l}\text { Data collection, analysis, } \\
\text { and interpretation; } \\
\text { revision of the } \\
\text { manuscript }\end{array}$ \\
\hline $\begin{array}{l}\text { Mateus } \\
\text { Mistieri } \\
\text { Simabukuro, } \\
\text { MD }\end{array}$ & $\begin{array}{l}\text { Universidade de São } \\
\text { Paulo, Faculdade de } \\
\text { Medicina, Hospital das } \\
\text { Clínicas }\end{array}$ & $\begin{array}{l}\text { Data collection, analysis, } \\
\text { and interpretation; } \\
\text { revision of manuscript }\end{array}$ \\
\hline
\end{tabular}

\section{References}

1. Arnaud L, Gorochov G, Charlotte F, et al. Systemic perturbation of cytokine and chemokine networks in Erdheim-Chester disease: a single-center series of 37 patients. Blood 2011;117:2783-2790.

2. Diamond EL, Dagna L, Hyman DM et al. Consensus guidelines for the diagnosis and clinical management of Erdheim-Chester disease. Blood 2014;124:483-492. 


\section{Neurology}

\section{Teaching Video NeuroImages: Multisystemic Erdheim-Chester Disease Presenting as a Cerebellar Ataxia}

Marcelo Houat de Brito, Marcos Castello Barbosa de Oliveira, Yuri Reis Casal, et al. Neurology 2021;96;e3005-e3006 Published Online before print February 16, 2021

DOI 10.1212/WNL.0000000000011714

This information is current as of February 16, 2021

\section{Updated Information \&} Services

References

Subspecialty Collections

Permissions \& Licensing

Reprints including high resolution figures, can be found at: http://n.neurology.org/content/96/24/e3005.full

This article cites 2 articles, 2 of which you can access for free at: http://n.neurology.org/content/96/24/e3005.full\#ref-list-1

This article, along with others on similar topics, appears in the following collection(s):

Gait disorders/ataxia

http://n.neurology.org/cgi/collection/gait_disorders_ataxia

Hematologic

http://n.neurology.org/cgi/collection/hematologic

MRI

http://n.neurology.org/cgi/collection/mri

Information about reproducing this article in parts (figures,tables) or in its entirety can be found online at:

http://www.neurology.org/about/about_the_journal\#permissions

Information about ordering reprints can be found online:

http://n.neurology.org/subscribers/advertise

Neurology ${ }^{\circledR}$ is the official journal of the American Academy of Neurology. Published continuously since 1951, it is now a weekly with 48 issues per year. Copyright @ 2021 American Academy of Neurology. All rights reserved. Print ISSN: 0028-3878. Online ISSN: 1526-632X.

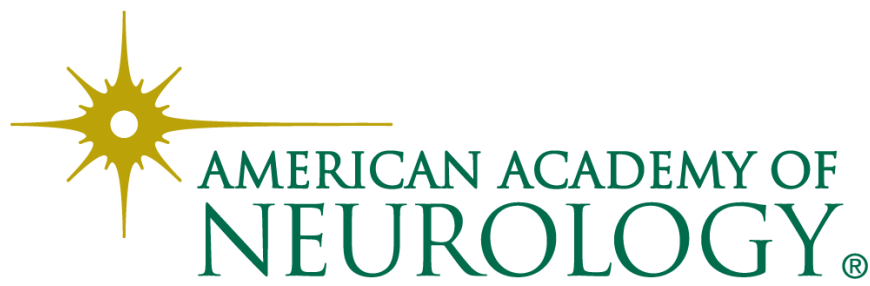

